\title{
Elevated Serum Reactive Oxygen Species Level Predicts Early Abortion
}

\author{
Joserizal Serudji', Nuzulia Irawati ${ }^{2}$, Johanes Cornelius Mose ${ }^{3}$, Hirowati Ali ${ }^{4}$, Yusrawati ${ }^{1}$ \\ ${ }^{1}$ Fetomaternal Division, Department of Obstetrics and Gynecology, Faculty of Medicine, Universitas Andalas, Padang, Indonesia \\ ${ }^{2}$ Parasitology Division, Faculty of Medicine, Universitas Andalas, Padang, Indonesia \\ ${ }^{3}$ Fetomaternal Division, Department of Obstetrics and Gynecology, Faculty of Medicine, Universitas Padjadjaran, Bandung, \\ Indonesia \\ ${ }^{4}$ Biochemistry Division, Faculty of Medicine, Universitas Andalas, Padang, Indonesia
}

Background: Impaired trophoblast invasion is associated with early abortion. The calorie needed for the trophoblast cell (TC) invasion is mainly met by adenosine triphosphate (ATP) produced in the mitochondria. Reactive oxygen species (ROS), byproduct of ATP synthesis, plays an important role in cellular physiology, but a high level of ROS may result in deoxyribonucleic acid (DNA) damage or cell dysfunction, thereby impaired TC invasion leading to early abortion. The study aims to determine elevated serum ROS level to predicts early abortion.

Materials and method: This was an observational study with a cross-sectional design. Fifty subjects with gestational age less than 12 weeks, consist of 25 early abortions and 25 normal pregnancies subjects, were included in this study. Clinical examination and diagnosis are carried out in 2 Hospitals and 5 Public Health Centers in Padang. Examination of ROS levels was carried out by enzyme-linked immunosorbent assay (ELISA) in the Biomedical Laboratory, Faculty of Medicine, Universitas Andalas. The Mann-Whitney test was used to analyze the difference of serum ROS levels, with a significance level of 0.05 .

Results: The subjects of the two study groups were equivalent in terms of age, gestational age, and gravidity $(p=0.051$, $p=0.453$, and $p=1.000)$. The median ROS levels were found to be $1.36(1.02-26.30) \mathrm{ng} / \mathrm{mL}$ in the early abortion and 1.20 $(0.43-2.75) \mathrm{ng} / \mathrm{mL}$ in the normal pregnancy $(p=0.003)$.

Conclusion: There is a significant difference between ROS levels in early abortion and normal pregnancy.

Keywords: ROS, early abortion, normal pregnancy

\section{Introduction}

The success of first trophoblast invasion determines the success of placentation, which also determines a good pregnancy outcome. The failure of trophoblast invasion is associated with unfavorable pregnancy outcomes such as spontaneous abortion, premature labor, preeclampsia, or stunted fetal growth. ${ }^{1}$ Trophoblast invasion requires high calories $^{2}$, which is met mainly from adenosine triphosphate (ATP) which is produced in the mitochondria. ${ }^{3}$

Date of submission: October 3, 2020

Last Revised: December 15, 2020

Accepted for publication: December 17, 2020

\section{Corresponding Author:}

Yusrawati

Fetomaternal Division, Department of Obstetrics and Gynecology

Faculty of Medicine, Universitas Andalas

Jl. Perintis Kemerdekaan, Padang 25127, Indonesia

e-mail: yusrawati65@med.unand.ac.id




ATP synthesis occurs through the process of oxidation phosphorylation. In addition to producing ATP, this process also produces reactive oxygen species (ROS). ROS which was initially considered only as a byproduct of ATP synthesis turned out to have an important role in regulating the physiological function of cells ${ }^{4}$, such as the processes of proliferation, differentiation, aging, regulation of transcription factors, inflammation, and various other regulatory functions. ${ }^{5}$ However, high levels of ROS can cause conditions of oxidative stress, which cause mitochondrial DNA damage (mutations and deletions), internal membrane damage, mitochondrial respiration disorders, which all of which lead to mitochondrial dysfunction and cell function disorders. ${ }^{3,4}$ Cell dysfunction causes impaired trophoblast invasion.

The association between ROS levels and impaired trophoblast invasion was concluded from studies on preeclampsia. The activity of superoxide dismutase (SOD), glutathione peroxidase (GPx), and 2,2'-azinobis (3-ethylbenzthiazoline-6-sulfonic acid) (ABTS) was significantly lower in preeclampsia. ${ }^{6}$ In preeclampsia, there is an increase in oxidative stress and a decrease in antioxidant capacity. ${ }^{7}$ Superoxide levels are higher in preeclampsia than in normal pregnancy. ${ }^{8}$ Malondialdehide (MDA) levels are greatly increased and the levels of GPx and SOD are greatly decreased in preeclampsia. ${ }^{9}$ These findings suggest that there is a state of oxidative stress in preeclampsia caused by high production of ROS or by the condition of unopposed ROS. Oxidative stress results in disruption of second wave trophoblast invasion which manifests as preeclampsia.

Unlike the second wave trophoblast invasion which requires normoxic conditions, the first wave of trophoblast invasion requires a hypoxic condition ${ }^{10}$, a condition that continues during the $1^{\text {st }}$ trimester. $^{11}$ Hypoxic conditions are a key factor for the proliferation, differentiation, and physiological invasion of trophoblasts, which in turn determine the success of placentation, growth, and development of pregnancy. ${ }^{10}$ On the other hand, hypoxic conditions can increase the production of ROS in oxidation phosphorylation, where the redox reaction shifts to hyperreduction due to lack of oxygen. ${ }^{12}$ High ROS levels have the potential to disrupt trophoblast invasion, and impaired trophoblast invasion in early pregnancy is associated with early abortion. High ROS levels are associated with the failure of second wave trophoblast invasion, which is clinically manifested as preeclampsia or fetal growth restriction. However, it is still unknown whether ROS levels are also associated with the failure of first-wave trophoblast invasion, which manifests clinically as early abortion. This study aims to prove the difference in ROS levels between early abortion and normal pregnancy. The analysis of these findings is expected to provide information on the role of ROS in impaired trophoblast invasion in early pregnancy.

\section{Materials and methods}

\section{Study Design}

This study was an observational one with a cross-sectional study design in trimester-1 pregnancy subjects (gestational age less than 12 weeks), comparing ROS levels between early abortion and normal pregnancy. Fifty cases of $1^{\text {st }}$ trimester pregnancy, consist of 25 cases of early abortion and 25 cases of normal pregnancy, at Dr. M. Djamil Hospital, Universitas Andalas Hospital, and 5 Community Health Centers in Padang City were involved in this study.

Subjects were classified to be "early abortion" if they had one of criteria as threatened abortion, inevitable abortion, incomplete abortion, or complete abortion. ${ }^{13}$ The study material was blood taken from vena cubiti. The ethical clearance was approved by the Research Ethics Committee Medical Faculty, Andalas University with ethical clearance number 363/KEP/FK/2018.

\section{Examination of ROS levels}

The material was blood taken from vena cubiti. Serum ROS levels were measured by enzyme-linked immunosorbent assay (ELISA), using the Bioassay Technology Laboratory kit Human ROS Modulator 1, (Catalogue No. E2134Hu, Bioassay Technology Laboratory, Shanghai, China) and the measurement results are expressed in units of $\mathrm{ng} / \mathrm{mL}$.

Examination of ROS levels was carried out firstly by preparing all reagents, samples, and standards. Sample and ELISA reagent were then added into each well, and incubated for 1 hour at $37^{\circ} \mathrm{C}$. After washing the plate 5 times, substrate solution $\mathrm{A}$ and $\mathrm{B}$ were added and incubated for 10 minutes at $37^{\circ} \mathrm{C}$. The stop solution was added, and resulting in the color development. We read the OD value within 10 minutes.

\section{Data Analysis}

The data analysis was conducted with SPSS version 21 (IBM Coorporation, Armonk, NY, USA). To test the ROS levels difference, we used was non-parametric MannWhitney test. 
Table 1. Subjects characteristics.

\begin{tabular}{lccc}
\hline \multirow{2}{*}{ Characteristic* } & Early Abortion & Normal Pregnancy & \multirow{2}{*}{$\boldsymbol{p}$-value } \\
\cline { 2 - 3 } & $(\mathbf{n = 2 5})$ & $(\mathbf{n = 2 5})$ & \\
\hline $\begin{array}{l}\text { Maternal age (years) } \\
\text { Mean } \pm \text { SD }\end{array}$ & $31.72 \pm 6.49$ & $28.60 \pm 4.23$ & 0.051 \\
\hline $\begin{array}{l}\text { Gestational age (weeks) } \\
\text { Mean } \pm \text { SD }\end{array}$ & $8.2 \pm 1.53$ & $7.8 \pm 2.16$ & 0.453 \\
\hline Gravidity & & & \\
Primigravida & $8(32 \%)$ & $8(32 \%)$ & 1.000 \\
Multigravidas & $17(68 \%)$ & $17(68 \%)$ & \\
\hline
\end{tabular}

*no case of recurrent abortion in both groups.

\section{Results}

\section{Subjects Characteristics}

The difference of characteristics between the early abortion group and normal preganancy group were shown in Table 1. There were no significant differences in the mean of age, mean of gestational age, and gravidity distribution between the two study groups $(p=0.51, p=0.453$, and $p=1.00$, respectively).

\section{Normality Test of ROS Levels Distribution}

The normality of ROS levels distribution was tested by the Saphiro-Wilk test, and the results were shown in Table 2. The ROS levels in the two study groups were not normally distributed, so to analyze the difference of ROS levels, Mann-Whitney test was used.

\section{Serum ROS Levels}

Table 3 showed that the median ROS levels in the early abortion group were significantly higher than those in the normal pregnancy group ( $p=0.003$ ), with median of the early abortion group was $1.36 \mathrm{ng} / \mathrm{mL}$ and median of the normal pregnancy group was $1.2 \mathrm{ng} / \mathrm{mL}$.

\section{Discussion}

The median ROS level was significantly higher in early abortion than that in normal pregnancy. The result showed

Table 2. The normality test of ROS levels distribution.

\begin{tabular}{lccc}
\hline \multicolumn{1}{c}{ Pregnancy } & statistic & df & $\boldsymbol{p}$-value \\
\hline Early abortion & 0.479 & 25 & 0 \\
Normal pregnancy & 0.846 & 25 & 0.001 \\
\hline
\end{tabular}

that high ROS levels were associated with early abortion. As previously explained, high levels of ROS causes oxidative stress, and oxidative stress causes mitochondrial dysfunction. ${ }^{3,4}$ Mitochondrial dysfunction causes oxidative phosphorylation to be suppressed so that more ROS is produced and calorie production decreases. Increasing levels of ROS further aggravate mitochondrial dysfunction, so that over time the ROS levels get higher and the calories produced decrease so that cell dysfunction gets worse. Cell dysfunction along with insufficient calories causes impaired trophoblast invasion, incomplete placentation, resulting in an early abortion.

The role of hypoxia in the incidence of early abortion is related to the fact that hypoxia causes a decrease in mitochondrial function as the main producer of calories. ${ }^{14}$ Mitochondria dysfunction caused trophoblast cells do not get enough calories. On the other hand, the main role of trophoblasts in the placentation process (i.e., invasion) requires high calories. ${ }^{15}$ ROS production can also be increased due to the direct effect of hypoxia, where the redox reaction shifts to hyper-reduction due to a lack of oxygen. ${ }^{12}$ The increased production of ROS due to hypoxia in early abortion needs to be considered because the blastocyst implants into the endometrium which is in a hypoxic state $e^{16}$, and this condition continues during the $1^{\text {st }}$ trimester. ${ }^{11}$ The reduced activity of complex-4 under hypoxic conditions slows the transfer of electrons through the electron transfer

Table 3. ROS levels in both study groups.

\begin{tabular}{lccc}
\hline \multicolumn{1}{c}{ Pregnancy } & $\begin{array}{r}\text { Median } \\
(\mathbf{n g} / \mathbf{m L})\end{array}$ & $\begin{array}{c}\text { Min-Max } \\
(\mathbf{n g} / \mathbf{m L})\end{array}$ & $\boldsymbol{p}$-value \\
\hline Early abortion & 1.36 & $1.02-26.30$ & 0.003 \\
Normal pregnancy & 1.2 & $0.43-2.75$ & \\
\hline
\end{tabular}


chain (ETC), increasing the transfer of unwanted electrons to molecular oxygen which results in a highly reactive superoxide anion. ${ }^{17}$ Electron release can be induced or inhibited by complexes I and III with specific inhibitors (such as rotenone and antimycin A), which cause high levels of ROS production in the mitochondria. Respiration inhibition and decreased mitochondrial membrane potential under anoxic or hypoxic conditions can stimulate ROS production. ${ }^{5}$

Hypoxia can also increase ROS production through increased miR-210 production. Hypoxia triggers an increase in miR-210 expression, both directly and through activation by hypoxia-inducible factor- $1 \alpha$ (HIF-1 $\alpha) \cdot{ }^{18,19}$ Upregulation of micro-RNA 210 (miR-210) represses iron-sulfur clusters $1 / 2$ (ISCU1/2), so that the activity of iron-sulfur clusters is disrupted, mitochondrial respiration is disrupted, which results in mitochondrial dysfunction. ${ }^{17,18}$

We concluded that oxidative stress plays a role in the pathophysiology of early abortion. This occurs because oxidative stress causes damage to trophoblast cells ${ }^{20}$, so that the first wave of trophoblast invasion process is disrupted, the placenta adhesion to the uterus is inadequate and eventually abortion occurs.

\section{Conclusion}

There is a significant difference between ROS levels in early abortion and normal pregnancy.

\section{References}

1. Velicky P, Knoefler M, Polheimer J. Function and control of human invasion tropjoblast subtypes: intrinsic vs maternal control. Cell Adh Migr. 2016; 10(1-2): 154-62.

2. Anun SA, Vince G, Quenby S. Trophoblast invasion. Hum Fertil. 2004; 7(3): 169-74.

3. Krumova K, Cosa G. Overview of reactive oxygen species. In: Nonell S, Flors C. Singlet Oxygen: Applications in Biosciences and Nanosciences, Volume 1. London: Royal Society of Chemistry; 2016. p.1-21.

4. Rabinovitch RC, Samborska B, Faubert B, Ma EH, Gravel S, Andrzejewski $\mathrm{S}$, et al. AMPK maintains cellular metabolic homeostasis through regulation of mitochondrial reactive oxygen Species. Cell Rep. 2017; 21(1): 1-9. doi: 10.1016/j. celrep.2017.09.026.
5. Angelova PR, Abramov AY. Functional role of mitochondrial reactive oxygen species in physiology. Free Radic Biol Med. 2016; 100: 81-5.

6. Chamy VM, Lepe J, Catalan A, Retamal D, Escobar J, Madrid EM. Oxidative stress is closely related to severity of preeclampsia. Biol Res. 2006; 39(2): 229-36.

7. Al-Kuarishy HM, Al-Gareeb AI, Al-Maiahy. Concept and connotation of oxidative stress in preeclampsia. J Lab Physicians. 2018; 10(3): 276-82.

8. Mannaerts D, Faes E, Cos P, Briedé JJ, Gyselaers W, Cornette J, et al. Oxidative stress in healthy pregnancy and preeclampsia is linked to chronic inflammation, iron status and vascular function. PLoS One. 2018; 13(9): e0202919. doi: 10.1371/journal.pone.0202919.

9. Shaikh SA, Vijayaraghavan R, Kumar DS, Manidip P. A comparative study of novel biomarkers on preeclampsia in relation to body mass index. Int J Res Pharm Sci. 2020; 11(1): 913-20.

10. Chakraborty D, Cui W, Rosario GX, Scott RL, Dhakal P, Renaud SJ, at al. HIF-KDM3A-MMP12 regulatory circuit ensures trophoblast plasticity and placental adaptations to hypoxia. Proc Natl Acad Sci USA. 2016; 113(46): E7212-21.

11. Wu F, Tian F and Lin Y, 2015. Oxidative stress in placenta: health and diseases. Biomed Res Int. 2015; 2015: 293271. doi: $10.1155 / 2015 / 293271$

12. Sasaki T, Awaji T, Simada K, Sasaki H. Increased levels of reactive oxygen species in brain slices after transient hypoxiainduced by a reduced oxygen supply. Neuropsychiatry. 2018; 8(2): 684-90.

13. Turrentine JE. Clinical Protocols in Obstetrics and Gynecology. London: Informa Healthcare; 2008

14. Golias T, Papandreou I, Denko NC. Hypoxic repression of pyruvate dehydrogenase activity is necessary for metabolic reprogramming and growth of model tumours. Sci Rep. 2016; 6: 31146. doi: 10.1038/srep31146.

15. Anin SA, Vince G, Quenby S. Trophoblast invasion. Hum Fertil. 2004; 7(3): 169-74.

16. Horii M, Li Y, Wakeland AK, Pizzo DP, Nelson KK, Sabatini K, et al. Human pluripotent stem cells as a model of trophoblast differentiation in both normal development and disease. Proc Natl Acad Sci USA. 2016; 113(27): E3882-91.

17. Thomas LW, Ashcroft M. Exploring the molecular interface between hypoxia-inducible factor signalling and mitochondria. Cell Mol Life Sci. 2019; 76(9): 1759-77.

18. Chan SY, Zhang YY, Loscalzo J. MicroRNA-210 controls mitochondrial metabolism during hypoxia by repressing the ironsulfur cluster assembly proteins ISCU1/2. Cell Metab. 2009; 10(4): $273-84$.

19. Guan Y, Song X, Sun W, Wang Y, Liu B. Effect of hypoxia-induced microRNA-210 expression on cardiovascular disease and the underlying mechanism. Oxid Med Cell Longev. 2019; 2019: 4727283. doi: $10.1155 / 2019 / 4727283$

20. Bilici M. The importance of oxidative stress in early week pregnancy loss. Crescent J Med Biol Sci. 2014; 1(4): 151-3. 